Original Article (short paper)

\title{
Physical education class can improve acute inhibitory control in elementary school students
}

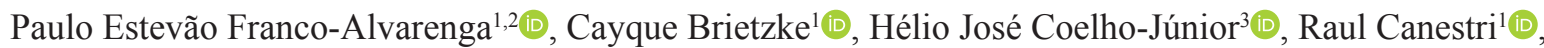 \\ Eduardo Yoshinori Nagata ${ }^{\mathbb{D}}$, Ricardo Yukio Asano ${ }^{1,4}{ }^{\mathbb{C}}$, Flávio Oliveira Pires ${ }^{1} \mathbb{0}$ \\ ${ }^{1}$ Universidade de São Paulo, USP, Exercise Psychophysiology Research Group, São Paulo, SP, Brazil; \\ ${ }^{2}$ Escola Superior de Educacao Fisica de Cruzeiro, ESEFIC, Cruzeiro, SP, Brazil; ${ }^{3}$ Universidade de \\ Campinas, Campinas, SP, Brazil; ; Universidade Ibirapuera, UNIB, São Paulo, SP, Brazil
}

\begin{abstract}
Aim: The aim of the study was to analyze the impact of a based-team sports physical education class on inhibitory control of elementary school children. Methods: Were selected 29 elementary school students (13 boys and 16 girls; $10.7 \pm 0.7$ years old; $43.2 \pm 8.2 \mathrm{~kg}$ weight; $147 \pm 5 \mathrm{~cm}$ height). Participants were submitted to a 50 -minute sports-based physical education class performed at moderate intensity. Inhibitory control was measured before and immediately after the end of the physical education class by the Stroop Test. Results: Results indicate that the variables derived from Stroop Test performance overall reaction time were significantly reduced after the physical education class $(p<0.05)$. Conclusion: These results suggest that a 50-minute sports-based physical education class performed at moderate intensity elicited a significant improvement on the inhibitory control elementary school students.
\end{abstract}

Keywords: stroop test, executive function, perceived exertion; children.

\section{Introduction}

Executive function (EF) includes the individual's capacity to identify and organize a purpose, create a strategy, evaluate the plan, identify new approaches if necessary, and perform the action ${ }^{1}$. Cognitive control is one of the EF components, operating in two distinct functions: proactive control and reaction control $^{1,2}$. Proactive control occurs before the stimuli action, being instructed to plan the action before it occurs ${ }^{2}$. In contrast, reaction control occurs in a way automatic and urgent, attending the aim of stimuli ${ }^{2,3}$. Thus, proactive control requires more cognitive recourse (i.e. memory, attention, focus, inhibitory control) and reaction control need to better responses of quick stimulus ${ }^{1,2}$.

Inhibitory control (IC) and selective attention are two components of $\mathrm{EF}^{4,5}$. These domains enable the individual to judge situations and make decisions in conflict situations ${ }^{4,5}$. IC can be divided into two subclasses: control attention (involves focus and selective attention) and cognitive inhibition (related to the ability to inhibit and select specific thoughts and memories) $)^{2}$. Therefore, in a school learning perspective, $\mathrm{EF}$ acts on cognitive control as IC is important to a successful scholar performance of children ${ }^{3,6}$.

In children, IC seems to be particularly important to school performance, since it is the capacity to constrain prepotent associations and/or previous and inappropriate ideas during the performance of several tasks ${ }^{1,7,8}$. Moreover, IC deficiency is considered a core factor in the aetiology of psychiatric disorders more prevalent in children associated with poor school performance, such as attention-deficit hyperactivity disorder
$(\mathrm{ADHD})^{9}$. Therefore, strategies to improve IC in children have been the focus of several studies ${ }^{10-12}$. For example, researches demonstrate the beneficial effects of physical activity on cognitive functioning ${ }^{10,11,13,14}$. Among children, recently published data has shown that acute aerobic exercise at moderate intensity (65-75\% heart rate $\left.\left[\mathrm{HR}_{\text {RESERVE }}\right]\right)$ improved IC measured immediately following the exercise session ${ }^{13}$.

Despite the importance of these researches, thus occurs in a laboratory environment so we do not know whether the same effect can occur in children's daily life. Because children enjoy playing games and sports, they may prefer recreational exercises than vigorous or moderate regular exercise programs ${ }^{15}$. Thus, as physical education class (PEC) is present as an exercise program in children's daily life and no studies have been designed to verify the effects of physical activity on EF in elementary school children, the propose of this study was analyzed the influence PEC on IC of elementary school children. We hypothesized that a moderate intensity PEC could increase temporarily the IC of elementary school children.

\section{Materials and Methods}

\section{Participants}

Twenty-nine (13 $\delta$ and 16 , between 10 and 12 years old) elementary school students $(13 \hat{\delta}, 10.7 \pm 0.7$ years old, $43.2 \pm 8.2 \mathrm{~kg}, 147 \pm 5 \mathrm{~cm}$ and $16 \circ, 10.3 \pm 0.8$ years old, 
$44.2 \pm 6.6 \mathrm{~kg}, 148 \pm 4 \mathrm{~cm}$ ) were selected from a private elementary school to participate in the present study. The sample size was determined using G-Power version 3.1. on the basis of a significance level at $5 \%$, a power $>0.8$, an effect size $(\mathrm{ES})>$ of 0.6 , and a loss of $20 \%$.

Eligibility criteria for this study were based on the absence of neither physical or intellectual disabilities, nor clinical, neuromotor, physiological, neurological, psychiatric, and cognitive impairments; have completed all experimental visits and measurements. The parents or legal custodians provided a written informed consent form. Students who were ineligible for the study participated in the PEC. However, they did not perform the Stroop Test. This study was approved by the Research Ethics Committee of the Faculdades Integradas Teresa D'Àvila (\#1.554.592) and was performed in accordance with the Declaration of Helsinki and Resolution 196/96 of the National Health Council.

\section{Procedure}

We requested to school's management for a quiet room that was used exclusively for anthropometric data collection, explanation of the procedures and application of the Stroop Test. Thus, in order to investigate the impact of a PEC on the
IC of elementary school children, participants were submitted to four visits interspersed by seven days, in the same period of the day. Visits were designed according to different aims: (a) In visit one, participants and their parents were familiarized with the researchers and signed the consent form; (b) in visit two, students were submitted to assessment of body composition and familiarized with the tools used in the present study (e.g., session rating of perceived exertion [SRPE] scale and Stroop Test). Thus, participants were familiarized with the modified Borg scale ${ }^{16}$ for children ${ }^{17}$ and the Stroop Test (see details below; 10 stimuli); (c) visit three was used as the control session, thus students individually performed the Stroop Test. Thereafter, they attended the PEC as configured in visit 4, thus familiarizing them with the Stroop Test procedures, PEC and SRPE session to be performed in visit 4. Importantly, only outcomes derived from the Stroop Test performed before the PEC were used in comparisons, thereby ensuring that eventual PEC-derived cross effects were controlled; (d) during the visit four, students performed the Stroop test before and immediately after the class. The Stroop test was performed on different visits (third and fourth) to avoid information bias. To ensure that the PECes were similar regarding the required physical effort, the SRPE was recorded and compared in both visit three and four. Figure 1 shows the study design used in the present study.

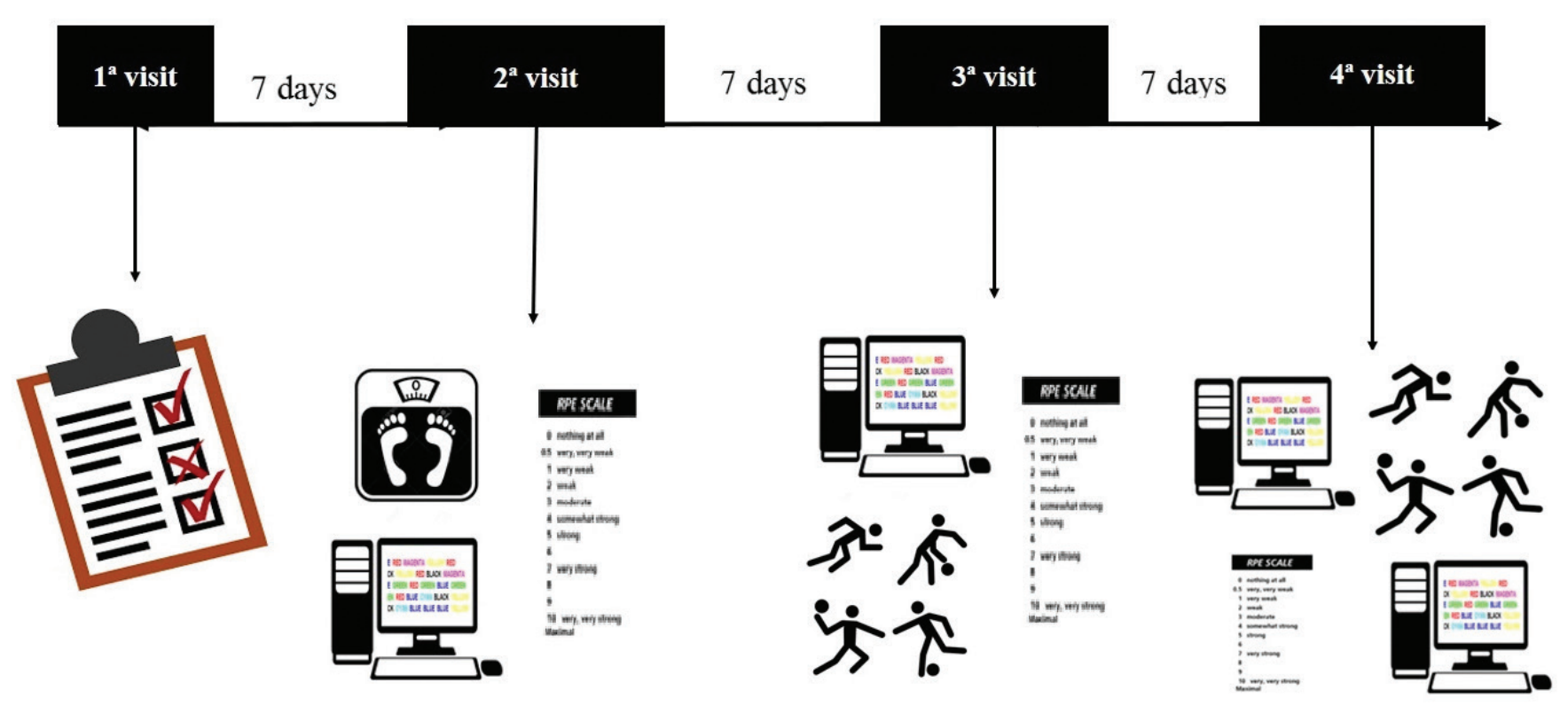

Figure 1. Experimental design Measures.

\section{Anthropometric measurements}

A weight-stadiometer scale (Filizola ${ }^{\circledR}$ ) was used to measure body mass $(\mathrm{kg})$ and height $(\mathrm{cm})$. Body mass index (BMI) was determined as the following:

$$
\frac{\mathrm{BMI}=(\mathrm{kg})}{(\text { height }[\mathrm{cm}])^{2}}
$$

\section{Physical Education Classes}

To provide adequate external validity, PEC was designed to represent a moderate intensity effort, comprising contents usually used in regular classes. As a warm-up, balls were made available for the students so that they had to throw the ball to other colleagues for 5 minutes. Then, they initiated the class performing an obstacle course activity for $\sim 15$ minutes, as a preparation part of 
a basketball tournament to be performed at the end of the current year. Thereafter, students played soccer while holding their hands for $\sim 15$ minutes, so that they had to keep the ball within the field game (they were continuously monitored in order to ensure constant displacement). Finally, they played dodgeball for the following 10 minutes (placed in opposite fields, they had to throw the ball towards the opponent so that students being touched by the ball changed to the other field as a penalty). Finally, students gave a walk around for the final 5 minutes as a cool-down. The classes took place in the normal class schedule (late afternoon, 4-6 PM), under the supervision of the charged teacher, with the temperature at the school ranging between $24-26^{\circ} \mathrm{C}$. The researchers remained near to the teacher, performed the data collection in the scheduled times, but without interfering directly in the class.

\section{Stroop Test}

The computerized version of the Stroop Test ${ }^{18}$ was used (Inquisit $\left.{ }^{\circledR}\right)^{13}$ in order to provide reaction time (total test time), time of response to every stimulus (congruent, incongruent and control), as well as successful and unsuccessful responses as outcomes. The test combines four colors (i.e. black, blue, green and red) with three different types of stimuli (i.e. congruent [word meaning corresponds to word color], incongruent [word meaning differs from word color] and control [indicate the color of an untitled monochrome square]). Stimulus was shown on a 15-inch monitor, and participants were required to press the answer corresponding to the color of the word. For example, if the word "red" was in "black", the correct response was "black".

\section{Session Rating of Perceived Exertion (SRPE)}

The rating of perceived exertion of PEC was quantified after class by the SRPE method ${ }^{19,20}$. The SRPE is strongly and linearly associated with the physical effort performed during the physical task and has been validated in the research literature ${ }^{21}$. Therefore, immediately after the end of the PEC (i.e., visits 3 and 4), participants were asked to indicating their SRPE on a modified Borg scale ${ }^{16}$ for children ${ }^{17}$.

\section{Statistics}

Data are presented as mean \pm standard deviation (SD). Homoscedasticity and distribution were tested through the Shapiro-Wilk's test. Stroop Test outcomes (i.e., overall reaction time, correct response, congruent, incongruent and control stimuli) were analyzed through a 2 × 2 repeated-design ANOVA having PEC (visit 3 vs visit 4) and moment (pre and post PEC) as fixed factors, while participants were the random one. Importantly, a repeated-ANOVA rather than a non repeated-ANOVA was used, due to the dependence relationship among multiple measures in a crossover, repeated-measures design. Importantly, only Stroop Test performance outcomes obtained before the PEC in control session were used, given the PEC-derived cross effects on cognitive performance. Thus, to make comparisons between visits 3 and 4 possible (i.e. control session vs PEC session), Stroop Test performance outcomes obtained before the PEC in control session were replicated as post PEC. Consequently, visit main effect (control session vs PEC session) and visit by moment interaction effect were reported, thereby making the report of moment main effect unnecessary. The multiple comparisons were corrected by Bonferroni test when F-values were significant. Furthermore, SRPE were analyzed through a t-student test between visit 3 and 4. Significance level was set at 5\% $(\mathrm{p}<0.05)$ (SPSS version 22; IBM, NY). Cohen's effect size $d$ was calculated to assess the magnitude of the results. The effect size (ES) was classified according to Hopkins, Marshall, Batterham, Hanin ${ }^{22}$ and defined as small $(<0.2)$, moderate $(0.2$ to 0.6$)$, large $(0.6$ to 1.2$)$, very large (1.2 to 2.0$)$, and extremely large $(>2.0)$.

\section{Results}

SRPE analyses indicated that there were no differences between the SRPE experienced in both PEC (visit three $=3.6 \pm 1.5 \mathrm{AU}$ vs visit four $=3.5 \pm 1.6 \mathrm{AU} ; \mathrm{p}>0.05)$, thus suggesting participants were submitted to similar effort levels during the classes.

Regarding the Stroop Test performance outcomes, there was no condition main effect $(\mathrm{p}=0.292 ; F=1.122 ; \mathrm{d}=0.283$, moderate ES) in correct answers (Figure 2 ). In contrast, we found differences in overall reaction time $(\mathrm{p}=0.000 ; F=95.394 ; \mathrm{d}=2.763$, extremely large ES), that reduces after PEC compared with control and before PEC $(p=0.000)$. Figure 2 and 3 depict these results.

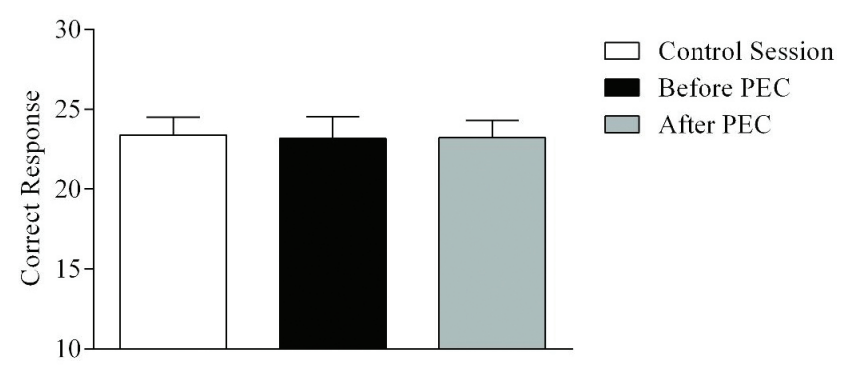

Figure 2. Results of correct response for control session (white bar), before PEC (black bar) and after PEC (gray bar). Only Stroop Test outcomes obtained before the PEC were reported.

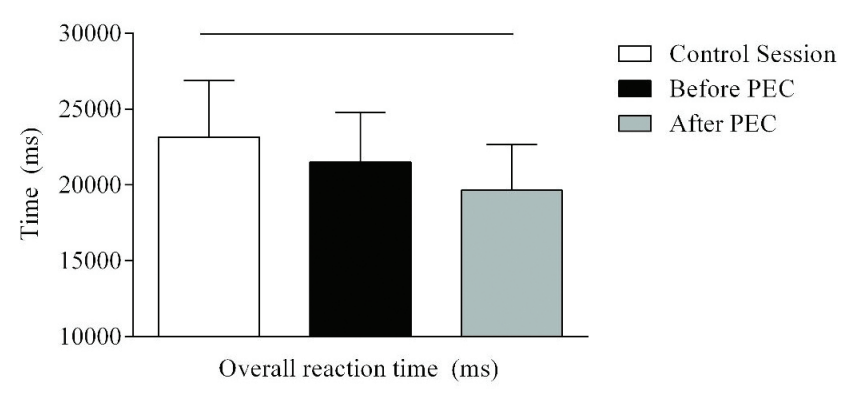

Figure 3. Results for overall reaction time in Stroop Test, for control session (white bar), before PEC (black bar) and after PEC (gray bar). Condition main effect was found $(p=0.000 ; F=95.394 ; d=2.763$, extremely large ES). Only Stroop Test outcomes obtained before the PEC were reported. 
Furthemore, PEC effects were observed in reaction time responses in congruent, incongruent and control stimuli. For example, congruent stimuli showed condition main effect $(\mathrm{p}=0.000 ; F=28.995 ; \mathrm{d}=1.439$, very large $\mathrm{ES})$ as reaction time reduced from pre to post PEC $(\mathrm{p}=0.000)$. Additionaly, condition main effect was further observed in incongruent stimuli ( $\mathrm{p}=0.000 ; F=33.155 ; \mathrm{d}=1.539$, very large ES), showing that $\mathrm{PEC}$ ameliorated reaction time responses after PEC compared with control and before PEC $(p=0.000)$. Finally, PEC reduces reaction time in control stimuli $(\mathrm{p}=0.000$; $F=72.689 ; \mathrm{d}=2.227$, extremelly large ES), precisally particpants were faster after PEC improve than other before class $(p=0.000)$. Figure 4 (panel A, B and C) depicts these reaction time responses.
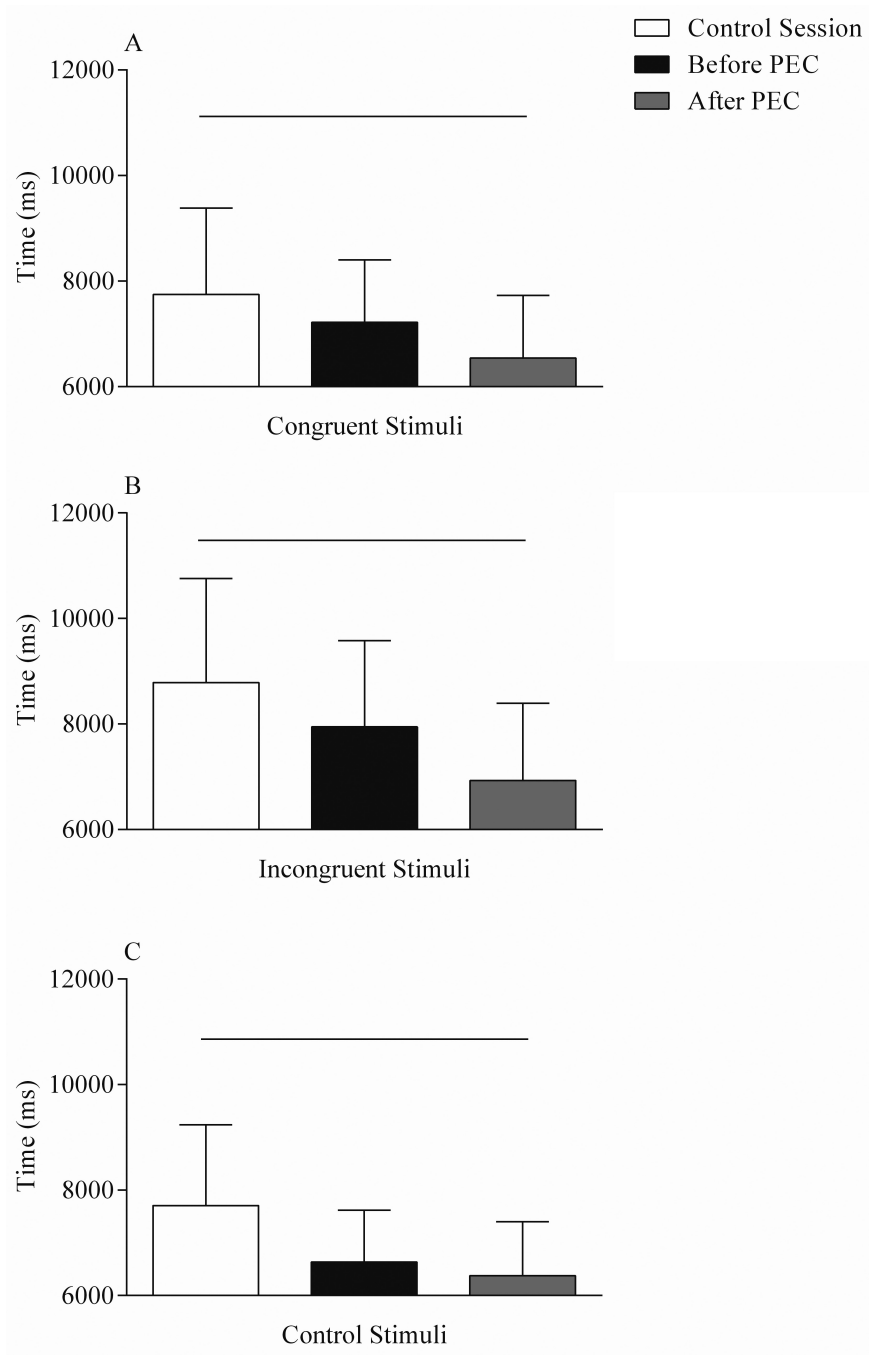

Figure 4. Results for reaction time for each stimuli in Stroop Test, for control session (white bar), before PEC (black bar) and after PEC (gray bar). Figure 4A showed reaction time for congruent stimuli $(\mathrm{p}=0.000 ; \mathrm{F}=28.995 ; \mathrm{d}=1.439$, very large ES). Figure $4 \mathrm{~B}$ showed reaction time for incongruent stimuli $(\mathrm{p}=0.000$; $\mathrm{F}=33.155 ; \mathrm{d}=1.539$, very large $\mathrm{ES})$. Figure $4 \mathrm{C}$ showed reaction time for control stimuli $(p=0.000 ; F=72.689 ; d=2.227$, extremely large ES. Only Stroop Test outcomes obtained before the PEC were reported.

\section{Discussion}

The present study investigated the effects of a PEC on the IC of elementary school students. Our findings indicate that a PEC was able to elicit significant improvements on IC, as we observed a decrease on the time to perform the cognitive test, followed by an improvement on reaction time responses to congruent, incongruent, and control stimulus.

Improvements on IC are particularly important, as IC is an important component of executive functioning and a key component of the cognitive function. In some of the psychiatric disorders more prevalent in children such as ADHD, a deficient IC may be considered as a central factor for impaired attention, focus, and concentration'. Moreover, intuitive actions derived from intuitive-heuristic systems have been suggested as a key aspect of cognitive bias (e.g., irrational, illogical and emotive thoughts and actions) and mental fixations (e.g., uncreative and biased behavior). In this regard, the IC is an important inhibitor for these behaviors as this possibly plays a key role in a successful problem-solving ${ }^{1,7}$.

A possible explanation for the results found in the present study is the increase of cerebral oxygenation after PEC. Several researchers have shown that exercise in moderate intensity increased the cerebral blood flow in areas of the prefrontal cortex, possibly as a consequence of the increased activation of the motor cortex during exercise ${ }^{23,24}$. In addition, Yanagisawa et al. ${ }^{25}$ showed that exercise in moderate intensity improves cognitive performance and increases activation of the left dorsolateral prefrontal cortex. Accordingly, results reported by a seminal study by Browne, Costa, Sales, Fonteles, Moraes, Barros ${ }^{13}$ showing a decreased reaction time after physical exercise in children.

Additionally, Peruyero, Zapata, Pastor, Cervelló ${ }^{26}$ studied 44 adolescents students in a Stroop Test after 3 different intensities (no exercise, moderate intensity, and vigorous exercise intensity) in PEC, having proposed for this class the "Zumba" dance as exercise. The results showed that vigorous intensity improved the number of correct responses in the Stroop Test when compared to moderate and no exercise intensity. In contrast with the present study, the individuals performed the Stroop Test on pencil-andpaper and thus failed to collect the reaction time. The authors suggested as an improvement for future studies, the application of the Stroop Test in a computer and the use of the perceived exertion for the exercise intensity control, thus both aspects have been done in the present study.

A meta-analysis demonstrated that exercise intensity may be a key factor to promote acute effects on cognitive performance ${ }^{23}$, as a recent investigation showed that moderate exercise intensity improved cognitive performance in students ${ }^{13}$. However, differences between the present study and Browne, Costa, Sales, Fonteles, Moraes, Barros ${ }^{13}$ may be related to the exercise intensity and exercise mode used in the physical activities. In this regard, although having a similar exercise mode between studies, we used intermittent exercises in the PEC, while Browne, Costa, Sales, Fonteles, Moraes, Barros 13 used continuous exercises. Briefly, participants performed $\sim 50$ minutes of moderate intensity PEC (according to the Borg's scale ${ }^{16}$ ) in the present study, while a moderate-to-vigorous intensity (i.e., 
$65-75 \% \mathrm{HR}_{\text {RESERVE }}$ ) was used by Browne, Costa, Sales, Fonteles, Moraes, Barros ${ }^{13}$. Additionally, a study by Browne, Costa, Sales, Fonteles, Moraes, Barros ${ }^{13}$ was conducted in a laboratory, thus probably limiting inferences to real-world situations due to the poor ecological validity, as performing PEC by using regular exercise on treadmills and cycle ergometers may be unrealistic for children. Furthermore, controlling sessions of PEC through the $65-75 \%$ of $\mathrm{HR}_{\text {RESERVE }}$ may be further unusual as it would require individualized orientation and apparatus for physiological and cardiac monitoring. In contrast, PEC based on playing through sports and games are more applied and motivational for children. Therefore, we suggest that an intervention that uses convention academic activities such as sports based in PEC may contribute to improving cognitive function required in other academic disciplines like math, history, grammar, etc. In fact, IC has been considered as an essential aspect to a successful academic achievement ${ }^{27}$.

Overall reaction time was different after the PEC when compared with control and before PEC, but there was no difference between control and measures performed before the PEC, thus confirming that there was no learning effect. When analyzing stimuli separately, there was a greater reduction in time for congruent and incongruent stimulus. These results are in agreement with the study of Sandroff, Hillman, Benedict, Mot128, who also found enhancement of IC in congruent and incongruent stimuli. Accordingly, these authors argued that the results are consequences to answer more difficult questions.

We further suggest that an intervention such as a sportsbased PEC contributes to better performance and understanding of other academic disciplines such as math, history, grammar, etc., since IC - combined with other aspects of EF - is essential to a successful academic achievement ${ }^{27}$. In conclusion, data of the present study suggest that a team-based PEC may play an important role to improve a short-term IC in elementary school students.

\section{Strengths and Limitations}

Prior studies have investigated the effect of physical exercise in the context of injuries, health, motor coordination, and social relations, however only a few have investigated the effects of physical education on cognitive parameters ${ }^{13,29}$. In this study, we evaluated the impact of a real PEC on IC responses of boys and girls. Despite the improved IC observed after performing a team-based PEC, some limitations should be highlighted. For example, due to the crossover characteristics of the present study, we have no control group constituted by different participants, since all subjects were submitted to all procedures, in an attempt to better represent the influence of the school PEC in the same subject. However, this may be a limitation from the point of view of possible learning in the Stroop test.

Another limitation is the absence of variations in PEC intensity (e.g., high, moderate, low). However, the study of Peruyero et. al..$^{26}$ evaluated the influence of different exercise intensities during PEC on IC results, their results showed the vigorous intensity was better to IC when compared with moderate intensity. Nevertheless, the authors monitored the exercise intensity across accelerometer and they suggested the use of session of rating perception effort to evaluate the intensity of PEC, in order to identify the real sensation of exertion of the subject after the exercise. Thus, future studies are required to extend the findings of the present study.

\section{References}

1. Diamond A. Executive functions. Annu Rev Psychol. 2013;64:135-68.

2. Braver TS. The variable nature of cognitive control: a dual mechanisms framework. Trends Cogn Sci. 2012 Feb;16(2):106-13.

3. Verburgh L, Königs M, Scherder EJA, Oosterlaan J. Physical exercise and executive functions in preadolescent children, adolescents and young adults: a meta-analysis. Br J Sports Med. 2014 Jun;48(12):973-9.

4. Bari A, Robbins TW. Inhibition and impulsivity: behavioral and neural basis of response control. Prog Neurobiol. 2013;108:44-79.

5. Diamond A. The early development of executive functions. Lifesp Cogn Mech Chang. 2006;70-95.

6. Gilmore C, Cragg L. Teachers' Understanding of the Role of Executive Functions in Mathematics Learning. Mind, Brain, Educ. 2014 Sep;8(3):132-6.

7. Cassotti M, Agogué M, Camarda A, Houdé O, Borst G. Inhibitory Control as a Core Process of Creative Problem Solving and Idea Generation from Childhood to Adulthood. New Dir Child Adolesc Dev. 2016 Mar;2016(151):61-72.

8. Medeiros W, Torro-Alves N, Malloy-Diniz LF, Minervino CM. Executive Functions in Children Who Experience Bullying Situations. Front Psychol. 2016/08/26. 2016;7:1197.

9. Shen IH, Tsai SY, Duann JR. Inhibition control and error processing in children with attention deficit/hyperactivity disorder: an event-related potentials study. Int J Psychophysiol. 2011/04/16. 2011;81(1):1-11.

10. Buck SM, Hillman CH, Castelli DM. The relation of aerobic fitness to stroop task performance in preadolescent children. Med Sci Sport Exerc. 2008;40(1):166-72.

11. Hillman CH, Castelli DM, Buck SM. Aerobic fitness and neurocognitive function in healthy preadolescent children. Med Sci Sport Exerc. 2005;37(11):1967-74.

12. Motes MA, Gamino JF, Chapman SB, Rao NK, Maguire MJ, Brier MR, et al. Inhibitory control gains from higher-order cognitive strategy training. Brain Cogn. 2014;84(1):44-62.

13. Browne RA, Costa EC, Sales MM, Fonteles AI, Moraes JF, Barros JF. Acute effect of vigorous aerobic exercise on the inhibitory control in adolescents. Rev Paul Pediatr. 2015/11/10. 2016;34(2):154-61.

14. Ludyga S, Gerber M, Brand S, Holsboer-Trachsler E, Pühse U. Acute effects of moderate aerobic exercise on specific aspects of executive function in different age and fitness groups: A meta-analysis. Psychophysiology. 2016 Nov;53(11):1611-26.

15. Calcaterra V, Larizza D, Codrons E, De Silvestri A, Brambilla P, Abela $\mathrm{S}$, et al. Improved metabolic and cardiorespiratory fitness during a recreational training program in obese children. J Pediatr Endocrinol Metab. 2013;26(3-4):271-6. 
16. Borg GA. Psychophysical bases of perceived exertion. Med Sci Sport Exerc. 1982/01/01. 1982;14(5):377-81.

17. Williams JG, Eston R, Furlong B. CERT: a perceived exertion scale for young children. Percept Mot Ski. 1994;79(3 Pt 2):1451-8.

18. Stroop JR. Studies of interference in serial verbal reactions. J Exp Psychol. 1935;18(6):643.

19. Day ML, McGuigan MR, Brice G, Foster C. Monitoring exercise intensity during resistance training using the session RPE scale. J Strength Cond Res. 2004;18(2):353-8.

20. Sweet TW, Foster C, McGuigan MR, Brice G. Quantitation of resistance training using the session rating of perceived exertion method. J Strength Cond Res. 2004;18(4):796-802.

21. Morris M, Dawes H, Howells K, Janssen R. Motor impairment and its relationship to fitness in children. BMJ Open. 2013;3(7).

22. Hopkins WG, Marshall SW, Batterham AM, Hanin J. Progressive statistics for studies in sports medicine and exercise science. Med Sci Sport Exerc. 2008/12/19. 2009;41(1):3-13.

23. Chang YK, Labban JD, Gapin JI, Etnier JL. The effects of acute exercise on cognitive performance: a meta-analysis. Brain Res. 2012/03/04. 2012;1453:87-101.

24. Endo K, Matsukawa K, Liang N, Nakatsuka C, Tsuchimochi H, Okamura H, et al. Dynamic exercise improves cognitive function in association with increased prefrontal oxygenation. J Physiol Sci. $2013 \mathrm{Jul} ; 63(4): 287-98$.

25. Yanagisawa H, Dan I, Tsuzuki D, Kato M, Okamoto M, Kyutoku Y, et al. Acute moderate exercise elicits increased dorsolateral prefrontal activation and improves cognitive performance with Stroop test. Neuroimage [Internet]. 2010;50(4):1702-10. Available from: http://www.ncbi.nlm.nih.gov/pubmed/20006719

26. Peruyero F, Zapata J, Pastor D, Cervelló E. The Acute Effects of Exercise Intensity on Inhibitory Cognitive Control in Adolescents. Front Psychol. 2017 May;8:921.
27. Alves CR, Tessaro VH, Teixeira LA, Murakava K, Roschel H, Gualano B, et al. Influence of acute high-intensity aerobic interval exercise bout on selective attention and short-term memory tasks. Percept Mot Ski. 2014;118(1):63-72.

28. Sandroff BM, Hillman CH, Benedict RHB, Motl RW. Acute effects of varying intensities of treadmill walking exercise on inhibitory control in persons with multiple sclerosis: A pilot investigation. Physiol Behav. 2016 Feb;154:20-7.

29. Etnier J, Labban JD, Piepmeier A, Davis ME, Henning DA. Effects of an acute bout of exercise on memory in 6th grade children. Pediatr Exerc Sci. 2014/07/15. 2014;26(3):250-8.

\section{Corresponding author}

Paulo Franco-Alvarenga 1000 Arlindo Béttio Av,. Ermelino Matarazzo, São Paulo (SP), Brazil Laboratory of Physical Activity Science, Postal Code 03828-000. Email: francope@usp.br

Manuscript received on October 17, 2018

Manuscript accepted on February 10, 2019

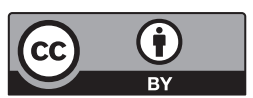

Motriz. The Journal of Physical Education. UNESP. Rio Claro, SP, Brazil - eISSN: 1980-6574 - under a license Creative Commons - Version 4.0 